\title{
COMPRIMIDOS REVESTIDOS POR PELÍCULA: TIPOS DE NÃO-CONFORMIDADES E SUAS CAUSAS
}

\author{
FILM-COATED TABLETS: NON-CONFORMITIES AND ITS CAUSES \\ COMPRIMIDOS RECUBIERTOS CON PELÍCULA: TIPOS DE NO \\ CONFORMIDADES Y SUS CAUSAS
}

Jaqueline Kalleian Eserian, Márcia Lombardo

Recebido em 24/07/2013, Aceito em 25/09/2014.

RESUMO: Os processos de compressão e revestimento de comprimidos são etapas críticas que interferem nas propriedades físicas e mecânicas do produto final. Com objetivo de descrever as características inerentes ao processo de revestimento por película e relacioná-lo com o aparecimento de não-conformidades no produto e suas possíveis causas, foi realizada a presente revisão bibliográfica. O uso de películas em comprimidos visa ações protetoras, estéticas e de modificação na taxa de liberação de princípios ativos. As forças da ligação interfacial entre a película e a superfície do comprimido e o estresse interno gerado dentro do revestimento são os fatores mais importantes que influenciam a adesão, sendo que a quantidade de revestimento aplicada e sua espessura são variáveis relevantes na obtenção de uma película adequada. Além de afetar a aparência, não-conformidades em comprimidos podem gerar consequências mais graves à saúde do paciente, relacionadas à perda de estabilidade do fármaco, alteração de sua liberação ou de propriedades organolépticas. A incidência de não-conformidades em comprimidos é um tema relevante para a indústria farmacêutica, portanto, faz-se necessário a realização de mais estudos que possibilitem estabelecer processos otimizados, protocolos para resolução de problemas e inclusão de novas tecnologias.

PALAVRAS CHAVES: Comprimidos; Polímeros; Tecnologia Farmacêutica; Controle de Qualidade. 
ESERIAN, J.K., LOMBARDO, M.

REF-ISSN1808-0804 Vol.XI (3),32-47, 2014.

ABSTRACT: Compression of tablets and film-coating process are critical events that influence physical and mechanical proprieties of the product. A bibliographical review was done with the objective of describing characteristics related to filmcoating process regarding its non-conformities and causes. Film-coating process is used for protection, esthetic appearance and modification in drug release rate. The most important factors that influence polymer adhesion are interfacial bonds between polymer and substrate and internal stress. The amount of coating and its thickness are important variables for obtaining a suitable film. Besides affecting product appearance, tablet non-conformities can be related to drug stability, alteration in drug release rate and organoleptical proprieties, with serious consequences for patients health. Tablet non-conformities incidence is a relevant problem for pharmaceutical industry, therefore, more studies are necessary in order to establish optimized processes, problem solving protocols and inclusion of new technologies.

KEY WORDS: Tablets; Polymers; Technology, Pharmaceutical; Quality Control.

RESUMEN: Los procesos de compresión y recubrimiento de comprimidos son pasos críticos que interfieren con las propiedades físicas y mecánicas del producto final. Con el fin de describir las características inherentes al proceso de recubrimiento con película y su relación con la ocurrencia de no conformidades en el producto y sus posibles causas, se realizó esta revisión de la literatura. El uso de películas en comprimidos pretende acciones de protección, estética y modificación en la velocidad de liberación de los ingredientes activos. La fuerza de los enlaces interfaciales entre la película y la superficie del comprimido y la tensión interna generada en el recubrimiento son los factores más importantes que influyen en la adhesión, y que la cantidad de recubrimiento aplicada y el espesor son variables relevantes en la obtención de una película adecuada. Además de afectar a la apariencia, no conformidades en comprimidos pueden causar más graves consecuencias para la salud del paciente, relacionadas a la pérdida de la estabilidad de la droga, modificación en su liberación o de las propiedades organolépticas del producto. La incidencia de no conformidades en los comprimidos es un tema relevante para la industria farmacéutica, por lo tanto, se hace necesario más estudios que permitan establecer procesos optimizados, protocolos para resolución de problemas y la inclusión de nuevas tecnologías. 
ESERIAN, J.K., LOMBARDO, $\mathrm{M}$.

REF-ISSN1808-0804 Vol.XI (3),32-47, 2014.

PALABRAS-CLAVE: Comprimidos; Polímeros; Tecnología Farmacéutica; Control de Calidad.

\section{INTRODUÇÃO}

O comprimido é considerado a forma farmacêutica mais comum, e sua obtenção se dá pelo confinamento radial de uma mistura de pós ou grânulos de substâncias ativas e excipientes em uma matriz, seguida de compactação axial por meio de punções. O processo de compressão envolve: o rearranjo das partículas; a compactação, que engloba os fenômenos de deformação elástica, deformação plástica, fragmentação de partículas e formação de ligações inter-particuladas; a descompactação e a ejeção. Trata-se de uma operação unitária crítica que interfere nas propriedades físicas e mecânicas do produto final e é influenciada por diversos fatores, como condições de processo, características de deformação (deformação elástica, visco-elástica, plástica e viscoplástica) e propriedades materiais dos componentes da formulação(1).

A compactação é considerada o estágio mais crítico do processo, devido ao fato da mistura passar por um estresse intenso à medida em que é comprimida até alcançar a pressão necessária para a descompactação. A descompactação ocorre quando os punções se separam, ocorrendo rápida queda da pressão conforme os mesmos se distanciam um do outro, com recuperação da tensão elástica acumulada durante 0 estágio de compressão(2).

Acredita-se que a taxa de recuperação elástica durante o estágio de descompactação seja um dos principais fatores responsáveis pela ocorrência de não-conformidades em comprimidos, sendo que quanto mais rápido ocorre, maior é a chance de falhas no processo. Outra possível causa de falha no processo é o aprisionamento de ar no interior do compactado. Embora ambas teorias pareçam ser razoáveis, poucas evidências foram obtidas na literatura científica até o presente momento ${ }^{(2)}$.

A utilização de comprimidos revestidos data de aproximadamente trinta anos atrás ${ }^{(3)}$, sendo atualmente bastante frequente na indústria farmacêutica. Revestimentos podem ser classificados como não-funcionais, também chamados de convencionais, quando o objetivo principal é a 
ESERIAN, J.K., LOMBARDO, M.

REF-ISSN1808-0804 Vol.XI (3),32-47, 2014.

proteção do princípio ativo e mudança da aparência do produto, ou funcionais, quando são utilizados com objetivo de modificar a liberação do

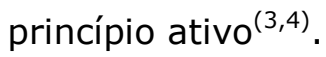

Estudos relacionados à área de desenvolvimento farmacotécnico possibilitam 0 planejamento de processos de fabricação reprodutíveis, com controle de produção, estabelecimento de especificações e obtenção de produtos de qualidade satisfatória.

Não-conformidades

relacionadas ao revestimento por película geram consequências como perda da tecnologia de fabricação desenvolvida para o produto, descaracterizando-o, além de comprometer a aderência do paciente ao tratamento, uma vez que o aspecto do medicamento é alterado.

Este trabalho teve como objetivo realizar uma revisão da literatura sobre a técnica de revestimento de comprimidos por película, com enfoque nos diferentes tipos de não-conformidades que os mesmos podem apresentar, bem como suas possíveis causas.

\section{METODOLOGIA}

A revisão bibliográfica foi realizada nas bases de dados Medline e ScienceDirect, utilizando os seguintes descritores nos idiomas português, inglês e espanhol: comprimidos revestidos por película, não-conformidades em comprimidos, revestimento de comprimidos, revestimento pelicular. Também foram consultados livros didáticos, dissertações e teses. Os critérios de inclusão compreenderam a abordagem direta do tema e disponibilidade eletrônica, sendo considerados estudos originais e de revisão, publicados entre 1975 a 2014. Foram excluídos estudos publicados fora do período estabelecido e estudos que não estavam diretamente relacionados ao tema. Após análise do material encontrado, foram selecionados 18 artigos sobre estudos relacionados à técnica de revestimento de comprimidos por película, sendo 13 deles diretamente relacionados a investigações sobre nãoconformidades em comprimidos. 
ESERIAN, J.K., LOMBARDO, M.

REF-ISSN1808-0804 Vol.XI (3),32-47, 2014.

\section{RESULTADOS/DISCUSSÃO}

Comprimidos revestidos

por película

A utilização de revestimentos por película em comprimidos é caracterizada pela deposição de uma camada de fina espessura contendo polímeros. O revestimento é aplicado sobre o núcleo em forma líquida, pulverizado por spray ${ }^{(5)}$. Durante a etapa de secagem, sob condições controladas, ocorre a evaporação do diluente do polímero.

A película tem como objetivos melhorar a estabilidade do princípio ativo por oferecer proteção contra agentes externos, como ar, umidade e luz; mascarar sabor e odor desagradáveis; oferecer textura lisa, facilitando a administração do medicamento; melhorar a aparência do comprimido; possibilitar uma rápida identificação do produto por parte do fabricante, dispensador e paciente, através da utilização de diferentes agentes de coloração; proporcionar maior resistência mecânica ao comprimido e assim facilitar 0 acondicionamento automático e minimizar a chance de contaminação cruzada por resíduos de pó por esfarelamento; melhorar a robustez do produto, uma vez que comprimidos revestidos são mais resistentes ao manuseio indevido (abrasão e atrito); desenvolver medicamentos de liberação modificada e entérica ${ }^{(5-7)}$.

A película é obtida pela dissolução ou dispersão de um polímero em solvente apropriado, que é pulverizado na forma de uma névoa fina sobre o núcleo, seguindo-se fenômenos físico-químicos até a formação do revestimento ${ }^{(7)}$, como mostra a Figura 1.
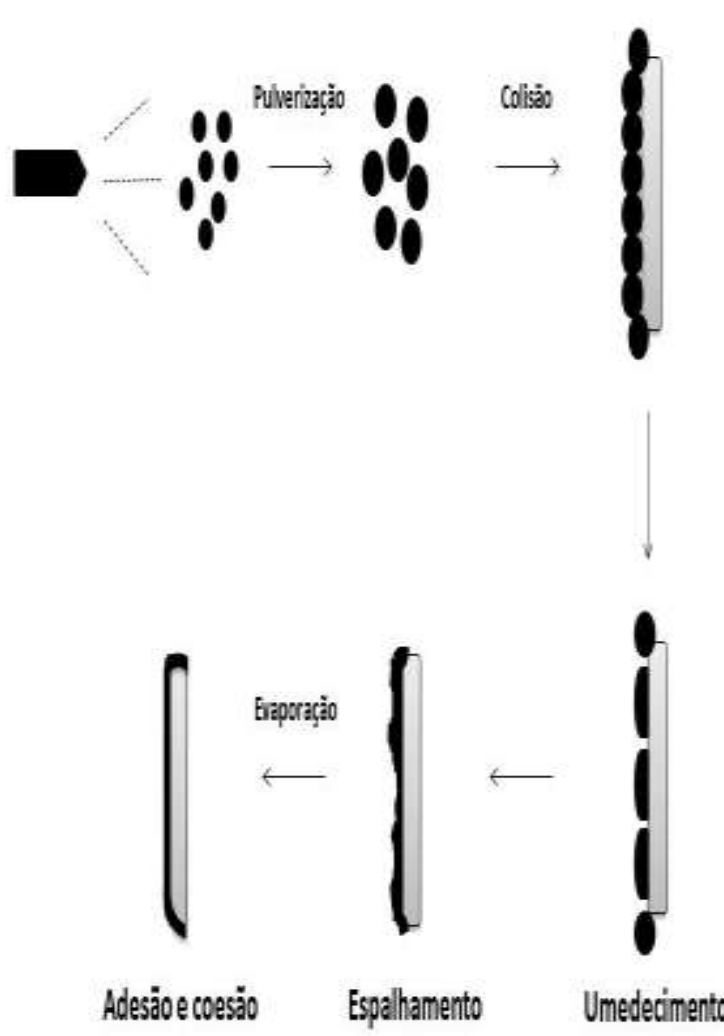

Figura 1: Desenho esquemático do processo de revestimento de comprimidos por película. Baseado em Felton, $2013^{(7)}$. 


\section{- Revista Eletrônica}

ESERIAN, J.K., LOMBARDO, M.

REF-ISSN1808-0804 Vol.XI (3),32-47, 2014.

Polímeros em solução formam películas após a evaporação do solvente, desde que as cadeias poliméricas estejam totalmente homogeneizadas. Polímeros em dispersão necessitam, além da evaporação do solvente, de coalescência das esferas individuais e interpenetração subsequente das cadeias individuais. Além disso, requerem uma temperatura mínima para a formação da película, sendo necessário, após o processo de revestimento, o armazenamento dos comprimidos em ambiente com temperatura e umidade controladas, a fim de garantir completa coalescência. A coalescência incompleta do polímero pode levar a mudanças significativas na liberação do princípio ativo ao longo do tempo ${ }^{(8)}$.

O revestimento por película geralmente apresenta uma espessura de 10 a $100 \mu \mathrm{m}$, sendo mais fino quando comparado com o revestimento com açúcar, utilizado na fabricação de drágeas. A escolha de um polímero com características de solubilidade desejáveis possibilita modificar o tempo de liberação do princípio ativo $^{(5,8)}$.
O processo de revestimento por película deve apresentar condições que permitam equilíbrio entre a quantidade de líquido a ser pulverizado e a secagem, além da uniformidade de distribuição sobre a superfície do núcleo.

A obtenção de revestimentos por película tem como pré-requisitos a boa adesão ao núcleo e a ausência de não-conformidades na película que afetem sua integridade e desempenho( ${ }^{(9)}$. As dificuldades do processo frequentemente estão relacionadas ao pequeno tamanho e falta de uniformidade na aspereza da superfície do núcleo ${ }^{(10)}$.

\section{Formulação de películas de revestimento}

As películas utilizadas no revestimento de comprimidos constituem-se principalmente de polímeros, agentes plastificantes e pigmentos $^{(11)}$, conforme ilustrado na Tabela 1. 


\section{- Revista Eletrônica de Farmácia}

ESERIAN, J.K., LOMBARDO, $\mathrm{M}$.

REF-ISSN1808-0804 Vol.XI (3),32-47, 2014.

\begin{tabular}{|c|c|c|}
\hline Polímeros & Agentes plastificantes & Pigmentos \\
\hline $\begin{array}{l}\text { Hidroxipropil-metilcelulose, } \\
\text { etilcelulose, hidroxipropil- } \\
\text { metilcelulose ftalato, acetato } \\
\text { ftalato celulose }\end{array}$ & $\begin{array}{c}\text { Glicerol, propilenoglicol, PEG } \\
400 \text {, dimetil ftalato, dietil ftalato, } \\
\text { dibutil ftalato, triacetina, óleo de } \\
\text { rícino }\end{array}$ & $\begin{array}{l}\text { Dióxido de titânio, óxido de ferro } \\
\text { vermelho, preto ou amarelo, } \\
\text { carbonato de cálcio }\end{array}$ \\
\hline
\end{tabular}

Tabela 1: Componentes utilizados na formulação de películas

Além de inerte e atóxico, um polímero ideal é aquele que apresenta solubilidade em diversos solventes e em $\mathrm{pH}$ de interesse do trato gastrointestinal; estabilidade na presença de luz, calor, umidade e ar; sabor, cor e odor aceitáveis e ausência de aderência ao toque ${ }^{(12)}$.

A função do solvente na formulação da película é transportar o polímero de maneira uniforme para a superfície do núcleo, sendo que o tipo de solvente utilizado pode influenciar o modo como a película é formada ${ }^{(12)}$. Quanto maior a interação polímerosolvente, maior a interação do polímero com a superfície do núcleo ${ }^{(10)}$.

As pontes de hidrogênio são o tipo de ligação mais comum entre a superfície do comprimido e o polímero. Ligações dipolo-dipolo e dipolo induzido também ocorrem, embora em menor extensão. Fatores que afetam o tipo ou a quantidade dessas ligações influenciam a adesão do revestimento ${ }^{(10)}$.

A utilização de polímeros de alto peso molecular resulta em películas mais resistentes, e quando uma rachadura é iniciada, sua propagação é bastante lenta. A adição de partículas maiores na formulação do revestimento reduz a incidência de rachaduras, assim como a variação na distribuição do tamanho de partículas $^{(13)}$.

Os agentes plastificantes aumentam 0 volume livre do polímero, tornando-o mais adaptável ao estresse gerado durante 0

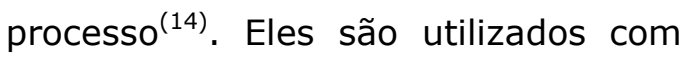
objetivo de melhorar as propriedades mecânicas do revestimento e facilitar a formação da película(10), gerando flexibilidade e elasticidade à mesma, o que consequentemente diminui sua fragilidade e a incidência de rachaduras.

Embora a água atue como um agente plastificante, pode causar inchaço no comprimido, à medida que se difunde durante $o$ período de 
ESERIAN, J.K., LOMBARDO, M.

REF-ISSN1808-0804 Vol.XI (3),32-47, 2014.

estocagem, causando estresses internos e enfraquecimento da interface previamente formada entre o revestimento e o núcleo. O armazenamento de comprimidos revestidos em ambientes com alto índice de umidade por períodos maiores que duas semanas resulta em falha do tipo coesiva, devido à absorção de água do ambiente ${ }^{(10)}$.

Excipientes utilizados no núcleo podem influenciar a adesão do revestimento por alterar a molhabilidade e o grau de ligação interfacial núcleo-película(10).

Os agentes lubrificantes melhoram a fluidez da mistura de pós ou granulado e facilitam a ejeção de comprimidos da matriz. Devido ao fato de apresentarem muitos grupos apolares, também podem influenciar na adesão do revestimento, dependendo de suas características e de sua concentração na formulação ${ }^{(10)}$.

Durante o teste de adesão, dois tipos de falha podem ocorrer. A falha adesiva resulta na remoção completa do revestimento contendo uma quantidade mínima de partículas provenientes do núcleo(10). A falha coesiva é caracterizada pela presença de partículas do núcleo na superfície do revestimento, ocorrendo quando as forças de ligação intermoleculares entre o revestimento e a superfície do núcleo são mais fortes do que entre o material particulado no interior do núcleo. Este tipo de falha é acompanhado pelo fenômeno de laminação, ou seja, separação em uma ou mais camadas.

A utilização de pigmentos gera a opacidade necessária para a proteção de componentes ativos fotossensíveis, além de auxiliar na identificação do produto pela cor. Embora em alguns casos a utilização de pigmentos seja necessária ${ }^{(5)}$, a inclusão de determinados tipos de pigmento na formulação exacerba o risco de ruptura do revestimento ${ }^{(14)}$, formando rachaduras ${ }^{(5)}$.

\section{Espessura e adesão de películas de revestimento}

A quantidade de revestimento aplicado ao núcleo e a espessura obtida determinam o aspecto, a opacidade, o grau de proteção contra a luz e umidade, e a modificação na taxa de liberação de princípios $\operatorname{ativos}^{(11)}$.

A falta de adesão do polímero ao núcleo pode levar ao acúmulo de umidade na interface revestimento- 
ESERIAN, J.K., LOMBARDO, M.

REF-ISSN1808-0804 Vol.XI (3),32-47, 2014.

núcleo, afetando significativamente a estabilidade de princípios ativos susceptíveis à degradação hidrolítica, além de comprometer a proteção mecânica do comprimido. Os fatores mais importantes que influenciam a adesão são as forças de ligação interfacial e o estresse interno gerado dentro do revestimento ${ }^{(10)}$.

As propriedades físicas e químicas do núcleo, principalmente a aspereza e a porosidade, interferem significativamente na adesão. A aspereza da superfície e a força de compressão utilizada alteram a área efetiva de contato da película, sendo que superfícies mais ásperas e irregulares são mais rapidamente molhadas, gerando maior contato interfacial. A quantidade e profundidade de penetração do líquido do revestimento também é um fator que pode influenciar $o$ contato interfacial, uma vez que comprimidos mais porosos propiciam uma penetração mais rápida.

Além disso, a formulação do revestimento pode oferecer características específicas de adesão. O uso de variados tipos de solventes, agentes plastificantes, antiaderentes, pigmentos e o próprio polímero estabelece diferentes interações interfaciais, fornecendo diferentes

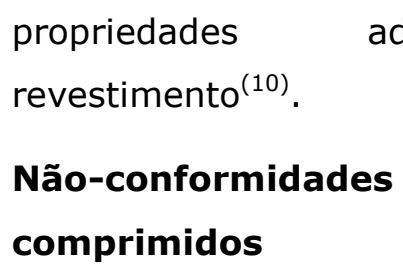

Diversos tipos de nãoconformidades em comprimidos podem ocorrer durante a produção. Alguns estudos mostram que existe um maior risco de incidência de nãoconformidades em comprimidos mais curvos, devido ao fato de apresentarem maior energia localizada no centro e extremidades, quando comparados a comprimidos planos $^{(15)}$.

Enquanto algumas nãoconformidades têm impacto somente na aparência do produto, outras podem afetar 0 perfil de administração do medicamento, comprometendo o tratamento do paciente.

De acordo com a literatura, as não-conformidades em comprimidos podem ser classificadas segundo a complexidade do problema e sua respectiva correção( ${ }^{(16)}$. A Tabela 2 mostra os tipos de não-conformidades que podem ser facilmente remediadas pela mudança de uma ou mais condições do processo, tais como ajustes da temperatura e da taxa de pulverização. 
ESERIAN, J.K., LOMBARDO, M.

REF-ISSN1808-0804 Vol.XI (3),32-47, 2014.

\begin{tabular}{|c|c|c|}
\hline Não-conformidade* & Definição & Possíveis causas \\
\hline $\begin{array}{l}\text { Blistering/Wrinkling }{ }^{(16)} \\
\text { (Bolhas) }\end{array}$ & $\begin{array}{l}\text { Destacamento local do } \\
\text { revestimento com } \\
\text { formação de bolha }\end{array}$ & $\begin{array}{l}\text { Aprisionamento de ar na camada de } \\
\text { revestimento ou entre o revestimento e o } \\
\text { núcleo, devido à alta temperatura durante a } \\
\text { pulverização; evaporação muito rápida } \\
\text { utilizando secagem em forno }\end{array}$ \\
\hline $\begin{array}{l}\text { Chipping } \\
\text { (Lascamento) }\end{array}$ & $\begin{array}{l}\text { Revestimento lascado ou } \\
\text { amassado, geralmente } \\
\text { nas bordas }\end{array}$ & $\begin{array}{c}\text { Alto grau de atrito no processo; punções } \\
\text { danificados; núcleos friáveis; falta de agentes } \\
\text { plastificantes }\end{array}$ \\
\hline $\begin{array}{l}\text { Erosion/Cratering }{ }^{(16)} \\
\quad \text { (Erosão) }\end{array}$ & $\begin{array}{l}\text { Revestimento com } \\
\text { crateras, expondo a } \\
\text { superfície do comprimido }\end{array}$ & $\begin{array}{c}\text { Taxa de pulverização muito alta; secagem } \\
\text { ineficiente; penetração do líquido no topo do } \\
\text { comprimido, devido sua maior porosidade, } \\
\text { com desintegração do núcleo e ruptura do } \\
\text { revestimento }\end{array}$ \\
\hline $\begin{array}{l}\text { Picking }{ }^{(6,12,16)} \\
\text { (Aderência) }\end{array}$ & $\begin{array}{l}\text { Destacamento local do } \\
\text { revestimento, expondo o } \\
\text { núcleo, após o } \\
\text { afastamento de } \\
\text { comprimidos previamente } \\
\text { aderidos (twinning) }\end{array}$ & $\begin{array}{l}\text { Taxa de pulverização muito alta; secagem } \\
\text { ineficiente }\end{array}$ \\
\hline $\begin{array}{l}\text { Pitting } \\
\text { (Buracos) }\end{array}$ & $\begin{array}{l}\text { Pequenos buracos, sem } \\
\text { rompimento visível do } \\
\text { revestimento }\end{array}$ & $\begin{array}{c}\text { Secagem em temperaturas maiores que o } \\
\text { ponto de fusão dos componentes do } \\
\text { revestimento }\end{array}$ \\
\hline
\end{tabular}

*Tradução livre para o português

Tabela 2: Não-conformidades que podem ser remediadas pelas condições do processo 
ESERIAN, J.K., LOMBARDO, M.

REF-ISSN1808-0804 Vol.XI (3),32-47, 2014.

\begin{tabular}{|c|c|c|}
\hline Não-conformidade* & Definição & Possíveis causas \\
\hline $\begin{array}{l}\text { Dulling/Blooming } \\
\text { (Opacidade) }\end{array}$ & $\begin{array}{l}\text { Revestimento opaco } \\
\text { imediatamente ou após o } \\
\text { armazenamento } \\
\text { prolongado em } \\
\text { temperaturas elevadas }\end{array}$ & $\begin{array}{l}\text { Acúmulo de componentes de baixo peso } \\
\text { molecular na superfície, principalmente } \\
\text { agentes plastificantes; processamento em } \\
\text { temperaturas muito altas, principalmente no } \\
\text { caso de polímeros de celulose em matrizes } \\
\text { aquosas; exposição à alta umidade }\end{array}$ \\
\hline $\begin{array}{l}\text { Blushing }{ }^{(16)} \\
\text { (Manchas } \\
\text { esbranquiçadas) }\end{array}$ & $\begin{array}{l}\text { Manchas esbranquiçadas } \\
\text { ou foscas geralmente em } \\
\text { revestimentos não- } \\
\text { pigmentados }\end{array}$ & $\begin{array}{l}\text { Precipitação do polímero pela utilização de } \\
\text { temperaturas inadequadas }\end{array}$ \\
\hline $\begin{array}{l}\text { Colour variation }{ }^{(16)} \\
\text { (Variação de cor) }^{\text {Varian }}\end{array}$ & $\begin{array}{l}\text { Variação de cor do } \\
\text { revestimento }\end{array}$ & $\begin{array}{l}\text { Homogeneização ineficiente; alteração da } \\
\text { frequência e duração da pulverização; } \\
\text { variação no tamanho e formato da zona de } \\
\text { pulverização }\end{array}$ \\
\hline $\begin{array}{l}\text { Infilling }^{(16)} \\
\text { (Preenchimento) }\end{array}$ & $\begin{array}{l}\text { Depósito de revestimento } \\
\text { na impressão em baixo } \\
\text { relevo, descaracterizando- } \\
\text { a }\end{array}$ & $\begin{array}{l}\text { Persistência de espuma no local da impressão } \\
\text { durante a pulverização devido ao menor atrito } \\
\text { existente, que impede a quebra da espuma. }\end{array}$ \\
\hline $\begin{array}{l}\text { Mottling } \\
\text { (Manchas) }\end{array}$ & $\begin{array}{l}\text { Presença de pontos mais } \\
\text { claros ou mais escuros } \\
\text { gerando distribuição } \\
\text { desigual de cor }\end{array}$ & $\begin{array}{c}\text { Mistura ineficiente de componentes de } \\
\text { diferentes cores; migração de pigmentos } \\
\text { solúveis em revestimentos do tipo aquoso; } \\
\text { formação de produtos de degradação } \\
\text { coloridos }\end{array}$ \\
\hline $\begin{array}{l}\text { Orange peel/Roughness } \\
\qquad(6,16) \\
\text { (Casca de laranja) }\end{array}$ & $\begin{array}{l}\text { Revestimento com } \\
\text { aparência áspera, similar } \\
\text { à casca de laranja, não- } \\
\text { lustroso }\end{array}$ & $\begin{array}{l}\text { Espalhamento inadequado do revestimento } \\
\text { devido à secagem prematura ou alta } \\
\text { viscosidade, geralmente combinado com alta } \\
\text { pressão de pulverização }\end{array}$ \\
\hline
\end{tabular}

*Tradução livre para o português

Tabela 3: Não-conformidades que podem ser remediadas pelas condições de processo e formulação do revestimento

No entanto, a resolução de algumas não-conformidades exige não só mudanças nas condições de processo, como também o

\section{Estresses internos e ocorrência de não-conformidades}

A proporção de estresses internos que se desenvolvem durante o processo de revestimento é dependente de diversos parâmetros aprimoramento da formulação da película, com substituição ou adição de excipientes ou aditivos (Tabela 3 ).

que envolvem tanto os componentes do revestimento quanto os componentes do núcleo. A escolha de excipientes que possuam coeficientes de expansão térmica similares minimiza 0 estresse interno, 


\section{i) Revista Eletrônica de Farmácia}

ESERIAN, J.K., LOMBARDO, M.

REF-ISSN1808-0804 Vol.XI (3),32-47, 2014.

favorecendo a adesão térmica(10).

Não-conformidades observadas

em comprimidos revestidos podem ser consequência do estresse gerado no interior do revestimento durante a etapa de secagem. À medida que a quantidade de diluente do polímero diminui por evaporação, o revestimento perde a habilidade de fluir, e devido à sua tendência de encolher, um estresse mecânico pode ser estabelecido ${ }^{(5)}$.

Quando temperaturas maiores que a temperatura ambiente são utilizadas na secagem, a força, elasticidade e adesão do revestimento podem ser afetadas. Conforme os comprimidos revestidos retornam à temperatura ambiente, um estresse térmico é formado devido à diferença entre os coeficientes de expansão térmica do revestimento e do núcleo, o que contribui para o aparecimento de rachaduras ${ }^{(5)}$.

0 estresse interno no revestimento também pode afetar a adesão da película. Revestimentos à base de celulose, pigmentados ou não, têm a adesão prejudicada com o armazenamento a $37^{\circ} \mathrm{C}$ e $75 \%$ de umidade relativa, provavelmente devido ao aumento do estresse interno causado por diferenças no coeficiente de expansão do revestimento e do comprimido, e também devido ao aumento do estresse de caráter volumétrico causado pelo inchaço do núcleo ${ }^{(10)}$. 


\section{i) Revista Eletrônica de Farmácia}

ESERIAN, J.K., LOMBARDO, $M$.

REF-ISSN1808-0804 Vol.XI (3),32-47, 2014.

\begin{tabular}{|c|c|c|}
\hline Não-conformidade* & Definição & Possíveis causas \\
\hline $\begin{array}{c}\text { Bridging of } \\
\text { intagliations }^{(6,9)} \\
\text { (Formação de } \\
\text { membrana) }\end{array}$ & $\begin{array}{l}\text { Formação de uma } \\
\text { membrana entre as } \\
\text { extremidades da } \\
\text { impressão em baixo } \\
\text { relevo, deixando-a } \\
\text { indistinta }\end{array}$ & $\begin{array}{l}\text { Estresses internos que superam as ligações } \\
\text { adesivas película-núcleo }\end{array}$ \\
\hline $\begin{array}{l}\text { Cracking/Splitting }{ }^{(6,12)} \\
\text { (Rachaduras) }^{\text {(Rach }}\end{array}$ & $\begin{array}{l}\text { Rachaduras pequenas e } \\
\text { finas no topo ou fundo } \\
\text { (cracking), ou próximas } \\
\text { às extremidades } \\
\text { (splitting) }\end{array}$ & $\begin{array}{c}\text { Utilização de polímeros de alto peso } \\
\text { molecular; estresses internos que excedem a } \\
\text { força de tensão; utilização de punções muito } \\
\text { côncavos que causam a rápida expansão dos } \\
\text { comprimidos }\end{array}$ \\
\hline $\begin{array}{l}\text { Peeling/Flaking } \\
\text { (Descascamento) }\end{array}$ & Descamação da película & $\begin{array}{l}\text { Erro na formulação; alto teor de umidade no } \\
\qquad \text { núcleo }\end{array}$ \\
\hline $\begin{array}{c}\text { Capping }^{(1,12)} \\
\text { (Descabeçamento) }\end{array}$ & $\begin{array}{l}\text { Separação total ou parcial } \\
\text { do topo ou fundo do } \\
\text { comprimido durante o } \\
\text { processamento, ejeção, } \\
\text { manuseio, teste físico, } \\
\text { envase, transporte ou } \\
\text { estocagem }\end{array}$ & $\begin{array}{c}\text { Recuperação elástica excessiva; alta } \\
\text { velocidade de compressão; propriedades } \\
\text { mecânicas anisotrópicas; aprisionamento de } \\
\text { ar durante a compressão e subsequente } \\
\text { expansão do comprimido na ejeção; } \\
\text { densidade heterogênea; pressão da matriz } \\
\text { causando tensão de cisalhamento no interior } \\
\text { do comprimido }\end{array}$ \\
\hline $\begin{array}{l}\text { Lamination }^{(12)} \\
\text { (Laminação) }^{\text {Lamina }}\end{array}$ & $\begin{array}{l}\text { Separação laminar em } \\
\text { uma ou mais camadas }\end{array}$ & $\begin{array}{l}\text { Aprisionamento de ar durante a compressão } \\
\text { com subsequente liberação do ar na ejeção }\end{array}$ \\
\hline
\end{tabular}

*Tradução livre para o português

Tabela 4: Não-conformidades resultantes de estresse interno.

As

não-conformidades

associadas com o elevado estresse

interno (Tabela 4) são mais críticas e

frequentemente requerem, além de mudanças nas condições de processo

e formulação da película, a reformulação do núcleo. 
ESERIAN, J.K., LOMBARDO, M.

REF-ISSN1808-0804 Vol.XI (3),32-47, 2014.

De acordo com a literatura, a espessura da película é diretamente proporcional ao quadrado do diâmetro em comprimidos planos, exercendo influência sobre a força de adesão ${ }^{(10,18)}$. Os limites de espessura para películas de revestimento são estabelecidos de acordo com a formulação, já que os componentes determinarão a adesão interfacial e a magnitude de estresses internos. Foi observado que a utilização de espessuras abaixo dos limites gera maior probabilidade de ocorrer laminação. Em geral, películas com espessura abaixo de $50 \mu \mathrm{m}$ tendem a apresentar bridging of intagliations ${ }^{(9)}$.

A inclusão de agentes plastificantes em formulações de películas é bastante prevalente, observando-se redução das forças intermoleculares entre as cadeias poliméricas e diminuição do estresse interno dentro do revestimento com o uso dos mesmos. Além disso, a inclusão deste tipo de componente

\section{CONSIDERAÇÕES FINAIS}

A incidência de nãoconformidades em comprimidos é um problema relevante para a indústria farmacêutica. As características físicas do produto podem refletir a qualidade do mesmo, sendo o aspecto o único resulta em diminuição da temperatura de transição vítrea do polímero, aumento da flexibilidade do filme e diminuição da incidência de rachaduras $^{(7)}$.

\section{Estudos apontam que a} inclusão de talco em revestimentos contendo ou não pigmentos reduz a incidência de rachaduras. A razão para este efeito é que o talco previne a geração de estresse interno durante o processo de secagem e promove o relaxamento no revestimento devido à forma, orientação e movimento de suas partículas ${ }^{(5)}$.

A otimização de formulações de películas prevê o aumento da concentração de agentes aglutinantes, a utilização de substâncias com propriedades de deformação plástica e a redução da porcentagem de partículas finas. Em termos de processamento, pode-se diminuir a penetração dos punções e a pressão de compressão, dentre outros ${ }^{(1)}$.

critério acessível ao consumidor para a checagem da qualidade do medicamento a ser administrado. Embora a boa aparência do medicamento não esteja necessariamente relacionada à sua segurança e eficácia, trata-se de um requisito primordial na aceitação do 
ESERIAN, J.K., LOMBARDO, M.

REF-ISSN1808-0804 Vol.XI (3),32-47, 2014.

consumidor.

somente o trabalho conjunto entre as

Além disso, as não-

áreas de desenvolvimento

conformidades em comprimidos

farmacotécnico, produção e controle

podem gerar consequências mais

graves, como dificuldade de

de qualidade, mas também a

administração, perda de proteção de

realização de mais estudos e o

ativos fotossensíveis, alteração na

empreendimento de novas técnicas

que visem 0 aprimoramento

taxa de liberação e modificação de

propriedades organolépticas.

Assim, faz-se necessário não

tecnológico e a diminuição da

incidência de não-conformidades em comprimidos revestidos por película.

\section{REFERÊNCIAS BIBLIOGRÁFICAS}

1. Akseli I, Ladyzhynsky N, Katz J, He X. Development of predictive tools to assess capping tendency of tablet formulations. Powder Technol. 2013;236:139-48.

2. Wu CY, Hancock BC, Mills A, Bentham AC, Best SM, Elliott JA. Numerical and experimental investigation of capping mechanisms during pharmaceutical tablet compaction. Powder Technol. 2008;181(2):121-29.

3. Bikiaris D, Koutri I, Alexiadis D, Damtsios A, Karagiannis G. Real time and nondestructive analysis of tablet coating thickness using acoustic microscopy and infrared diffuse reflectance spectroscopy. Int. J. Pharm. 2012;438(1-2):33-44.

4. Kucera SA, Felton LA, McGinity JW. Physical aging in pharmaceutical polymers and the effect on solid oral dosage form stability. Int. J. Pharm. 2013;457(2):42836.

5. Gibson SHM, Rowe RC, White ETF. Mechanical properties of pigmented tablet coating formulations and their resistance to cracking I. Static mechanical measurement. Int. J. Pharm. 1988;48(1-3):63-77.

6. Troy DB. Remington: The science and practice of pharmacy. 21.ed. Baltimore: Lippincott USA; 2005.

7. Felton LA. Mechanisms of polymeric film formation. Int. J. Pharm. 2013;457(2):423-7.

8. Rowe RC. Tablet-tablet contact and mutual rubbing within a coating drum - an important factor governing the properties and appearance of tablet film coatings. Int. J. Pharm. 1988;43(1-2):155-9.

9. Rowe RC. Film/tablet adhesion, film thickness, internal stresses and bridging of the intagliations-A unified model with practical implications. Eur. J. Pharm. Sci. 2007;30(3-4):236-9.

10. Felton LA, McGinity JW. Adhesion of polymeric films to pharmaceutical solids. 


\title{
- Revista Eletrônica
}

\author{
ESERIAN, J.K., LOMBARDO, M. \\ REF-ISSN1808-0804 Vol.XI (3),32-47, 2014.
}

Eur. J. Pharm. Biopharm. 1999;47(1):3-14.

11. Rowe RC. Predicting film thickness on film coated tablets. Int. J. Pharm. $1996 ; 133(1-2): 253-6$.

12. Kasture PV, Parakh SR, Hasan SA, Gokhale SB. Pharmaceutics - I: First year diploma in pharmacy. 15. ed. Pune: Nirali Prakashan; 2008.

13. Rowe RC, Roberts RJ. The effect of some formulation variables on crack propagation in pigmented tablet film coatings using computer simulation. Int. J. Pharm. 1992;86(1):49-58.

14. Gibson SHM, Rowe RC, White ETF. The mechanical properties of pigmented tablet coating formulations and their resistance to cracking II. Dynamic mechanical measurement. Int. J. Pharm. 1989;50(2):163-73.

15. Fassihi AR, Parker MS. Formulation effects on capping tendencies. Int. J. Pharm. $1986 ; 31(3): 271-3$.

16. McGinity JW. Aqueous Polymeric Coatings for Pharmaceutical Dosage Forms Drugs and the Pharmaceutical Sciences. 3.ed. New York: New York USA; 2008.

17. Rowe RC, Forse SF. Pitting - a defect on film-coated tablets. Int. J. Pharm. $1983 ; 17(2-3): 347-9$.

18. Fisher DG, Rowe RC. The adhesion of film coatings to tablet surfacesinstrumentation and preliminary evaluation. J. Pharm. Pharmacol. 1976;28(12):886-9. 